\title{
Thermal invisibility through geometrical transformation: a segmented cloaking approach
}

\author{
E. H. Ooi \& V. Popov \\ Environmental and Fluid Mechanics, Wessex Institute of Technology, \\ Ashurst, Southampton, UK
}

\begin{abstract}
Thermal invisibility through geometrical transformation can be achieved; however, the required materials would be inhomogeneous and thermally anisotropic. The difficulty in manufacturing materials that are inhomogeneous and thermally anisotropic in the desired way, has been a major obstacle in the fabrication of a thermal cloaking device. In this study, the potential of using segmented cloaking to mimic the inhomogeneous and thermally anisotropic properties to achieve thermal invisibility is explored. Segmented cloaking involves the use of a multi-layered structure - each layer defined by homogeneous and thermally isotropic materials - to replace the inhomogeneous and anisotropic material. This reduces the requirements on the materials used in the fabrication of a thermal cloaking device. The effectiveness of the segmented thermal cloak is examined numerically and the effects of the number of segments used and the thickness ratio of the segments on the level of thermal invisibility achieved are investigated.
\end{abstract}

Keywords: segmented thermal cloaking, coordinate transformation, thermal resistance, homogeneous and isotropic, thermal conductivity.

\section{Introduction}

Pendry et al. [1] demonstrated that invisibility from an electromagnetic field can be achieved by bending the trajectory of the electromagnetic waves around a given object. The diversion of the electromagnetic waves is carried out mathematically through a linear geometrical transformation, which in practice, leads to the use of inhomogeneous and anisotropic materials as the cloaking 
material. The same geometrical transformation may be applied to the thermal field to achieve thermal invisibility [2-4]. In this case, the propagation of heat flux is diverted around an object such that it becomes thermally invisible to an observer.

One of the major obstacles in manufacturing a thermal cloaking device based on geometrical transformation is the high requirements in fabricating materials that are inhomogeneous and thermally anisotropic. The use of metamaterials artificially created materials that have properties not found in nature - has led to the successful fabrication of an electromagnetic wave cloaking device in the microwave range [5]. However, metamaterials which could be tailored to achieve thermal invisibility have not been reported yet.

In this study, the use of a multi-segmented structure to mimic the inhomogeneous and thermally anisotropic materials required to achieve thermal invisibility is explored. This approach is known as segmented cloaking, which involves the discretization of the cloaking region into segments - each segment comprises of materials with homogeneous and isotropic thermal properties - to mimic the overall inhomogeneous and anisotropic properties of the thermal cloak. Segmented cloaking offers a more realistic approach in fabricating thermal cloaks, since the use of homogeneous and thermally isotropic materials reduces the requirement on the materials.

\section{Geometrical transformation}

Consider the steady-state heat conduction equation:

$$
\nabla \cdot(k \nabla T)=0,
$$

which may be expressed in terms of the heat flux vector, $\mathbf{q}$ :

$$
\begin{aligned}
& \nabla \cdot \mathbf{q}=0, \\
& k \nabla T=\mathbf{q} .
\end{aligned}
$$

The foundation behind thermal invisibility through geometrical transformation is that the governing equation, represented here by the heat conduction equation in (2), is invariant under coordinate transformation. In other words, (2) retains the same form when mapped onto a new coordinate system [2-4].

In the new coordinate system, the thermal conductivity is replaced by one that is inhomogeneous and anisotropic [2-4], i.e.

$$
\kappa=\mathbf{H}^{-1} \mathbf{g}^{-1} \mathbf{h}\left|e_{1} \cdot\left(e_{2} \times e_{3}\right)\right| k,
$$

where $\kappa$ is the modified thermal conductivity, $e_{1}, e_{2}$ and $e_{3}$ are base vectors in the transformed coordinates and the coefficients $\mathbf{H}, \mathbf{g}$ and $\mathbf{h}$ are coefficients associated with the transformation. 
For simplicity, the thermal cloak is assumed to be an infinitely long cylinder with inner radius $R_{1}$ and outer radius $R_{2}$. The transformation that diverts the heat flux from the region $r<R_{2}$ to the compressed space of $R_{1}<r<R_{2}$ is given by [1]

$$
r^{\prime}=R_{1}+\frac{r\left(R_{2}-R_{1}\right)}{R_{2}}, \quad \theta^{\prime}=\theta, \quad z^{\prime}=z .
$$

where $r, q$ and $z$ are the coordinates in the cylindrical coordinate system. Applying the linear geometrical transformation in (4), the modified thermal conductivity in (3) is obtained as

$$
\kappa_{r}=\frac{r^{\prime}-R_{1}}{r^{\prime}}, \quad \kappa_{\theta}=\frac{r^{\prime}}{r^{\prime}-R_{1}}, \quad \kappa_{z}=\left(\frac{R_{2}}{R_{2}-R_{1}}\right)^{2} \frac{r^{\prime}-R_{1}}{r^{\prime}} .
$$

\section{Segmented cloaking}

Segmented cloaking is carried out by using a two-step process. In step 1, the inhomogeneous and thermally anisotropic material is discretized into $M$ segments, each assuming homogeneous and anisotropic properties and can be of different thickness. In step 2, each segment obtained from step 1 is partitioned into two sublayers - each sublayer is homogeneous and thermally isotropic. This is shown in Figure 1. In this case, the homogeneous and isotropic thermal conductivity of each sublayer is determined using [4]

$$
\begin{aligned}
& k_{A}=\frac{-\left[\kappa_{\rho} F-\kappa_{\theta} G\right]+\sqrt{\left[\kappa_{\rho} F-\kappa_{\theta} G\right]^{2}-4 \eta^{2} G \kappa_{\rho} \kappa_{\theta}}}{2 \eta(1+\eta)}, \\
& k_{B}=\frac{-\left[\kappa_{\rho} F-\kappa_{\theta} G\right]-\sqrt{\left[\kappa_{\rho} F-\kappa_{\theta} G\right]^{2}-4 \eta^{2} G \kappa_{\rho} \kappa_{\theta}}}{2 \eta(1+\eta)}
\end{aligned}
$$

where $F=\left(1-\eta^{2}\right), G=(1+\eta)^{2}, k_{a}$ and $k_{b}$ are the thermal conductivities of sublayers 'A' and ' $\mathrm{B}$ ', respectively and $\eta$ is the thickness ratio such that $\eta=d_{B} / d_{A}$. The '+' and ' - ' signs before the square root in (6) indicate that sublayer ' $A$ ' is of higher thermal conductivity than sublayer ' $B$ '.

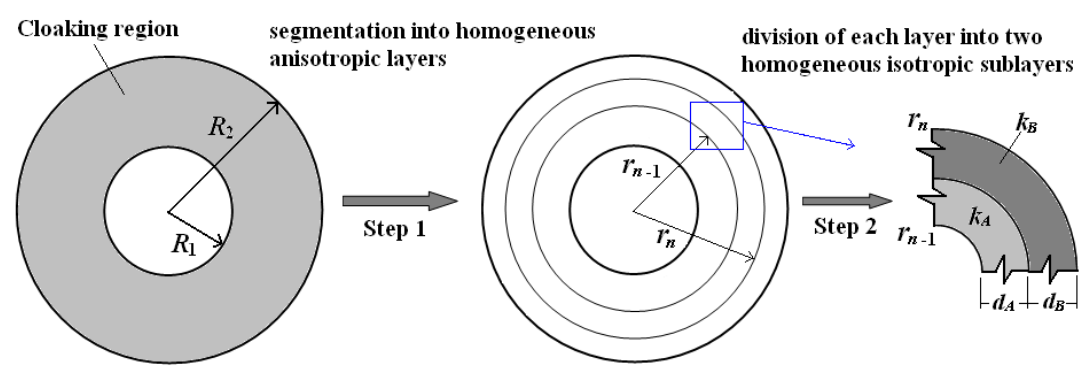

Figure 1: $\quad$ Two-step process of segmented cloaking. 
For simplicity, the segmented thermal cloak is hereafter referred to as the $M$ segmented cloak, with $M$ denoting the number of segments obtained from the first step in the segmentation process. For instance, the 2-segmented cloak refers to the segmented thermal cloak with 2 segments. Note that the total number of segments is $2 M$ after the second segmentation procedure. The single layer thermal cloak with inhomogeneous and thermally anisotropic properties is referred to as the ideal cloak.

\section{Numerical results}

\subsection{Ideal thermal cloak}

Numerical simulations were carried out in the dimensionless variables. To illustrate the effectiveness of segmented cloaking in mimicking the inhomogeneous and thermally anisotropic properties of the thermal cloak, an infinitely long cylindrical cloak with $R_{1}=0.1$ and $R_{2}=0.2$ was considered. Only the circular cross-section of the cylinder was simulated due to symmetry in the $z$ direction. The cloak was positioned at the centre of a square of dimensions $0.3 \times 0.3$. Temperatures of 0 and 1 were specified on the left and right hand side vertical walls. The horizontal top and bottom walls were thermally insulated. All the models were solved using the finite element method implemented with the commercial software COMSOL Multiphysics 4.2.

Figure 2(a) plots the contours of temperature and the isotherms inside the solution domain for the inhomogeneous and anisotropic thermal cloak, hereafter referred to as the ideal cloak. The bending of the isotherms around the region $r=R_{1}$ is clearly depicted. The isotherms to the left of the ideal cloak were identical to those on the right. This suggests that any object placed within the cloaked region of $r<R_{1}$ will become thermally invisible to an observer situated to the left of the cloak. Another distinct feature of the thermal profile in Figure 2(a) is the uniform temperature within the cloaked region.

\subsection{Effects of number of segments in the segmented thermal cloak}

Three cases of segmented thermal cloaks were considered, i.e. for $M=2,10$ and 20. The contours of temperature and the isotherms are plotted in Figures 2(b), 2(c) and 2(d). With only two segments, the bending of the heat flux around the region $r=R_{1}$ was less effective, which indicates a lower degree of thermal invisibility. The presence of isotherms inside the cloaked region also indicates a significant thermal gradient.

The effectiveness of the segmented cloaks increased with the number of segments used. From Figure 2, one may observe that the thermal profile and the isotherms of the 20-layers segmented cloak match closely those of the ideal cloak. In general, the range of thermal conductivity within the cloaking region $R_{1}<r<R_{2}$, increase with the number of segments used. This leads to a smoother propagation of heat flux across the interface between two adjacent sublayers. 

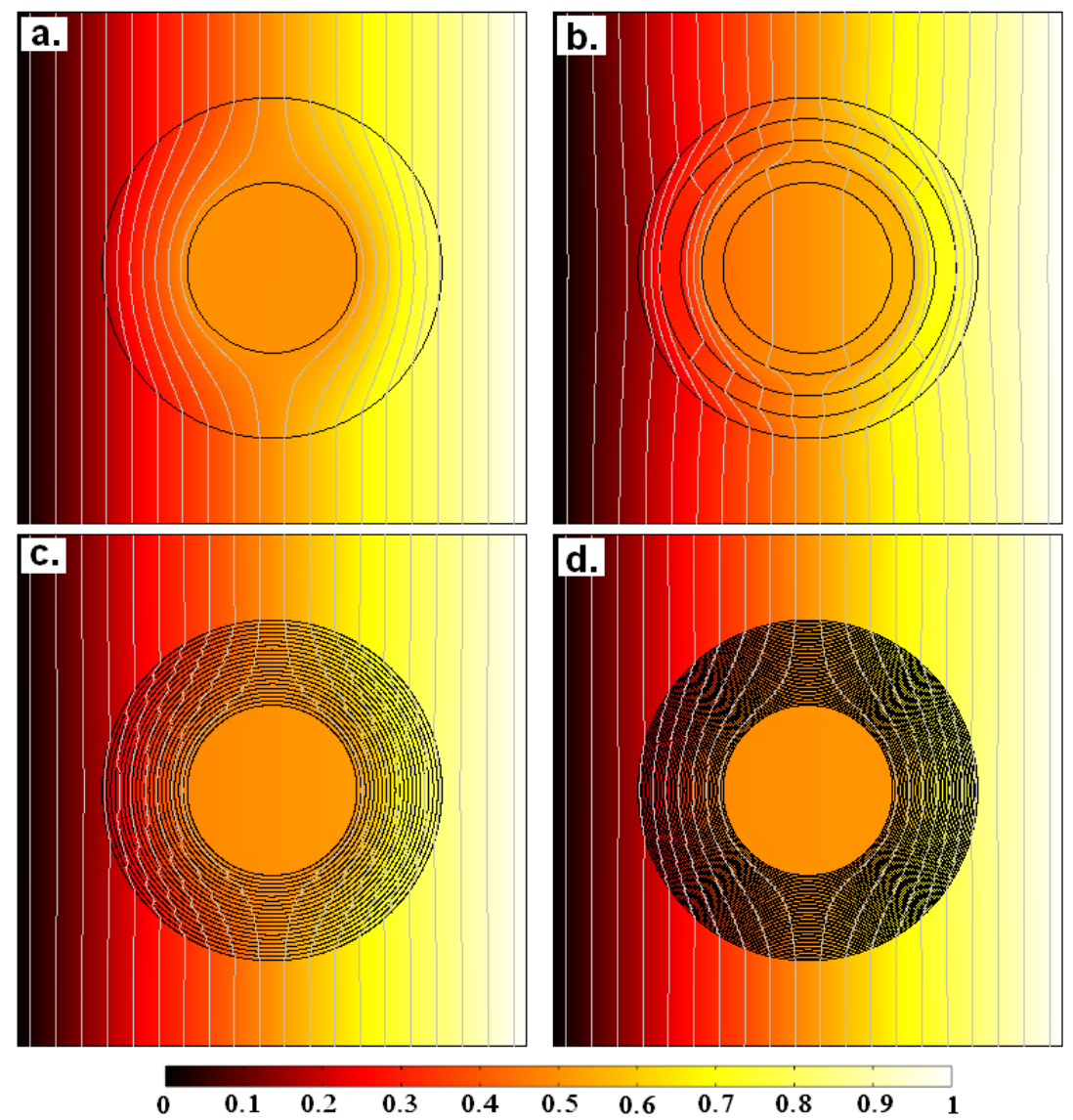

Figure 2: Contours of temperature and isotherms of the (a) ideal cloak, (b) two-layers, (c) 10-layers and (d) 20-layers segmented cloak.

Insufficient number of segments yielded jagged-like isotherms as shown in Figure 2(b) for the 2-layers segmented cloak.

Figure 3 plots the temperature profile along the geometrical centre of the square region, i.e. along the axis $y=0$. The profile for the case without thermal cloaking is also shown, which is indicated by the diagonal line. With the 2-layers segmented cloak, the temperature profile showed significant deviation from the case of the ideal cloak. The discrepancy in the temperature profile at $-0.3<\mathrm{x}<-0.2$ between the 2-layers segmented cloak and the case without thermal cloak suggests poor thermal cloaking. The effectiveness of the segmented cloak can be improved by increasing the number of layers. 


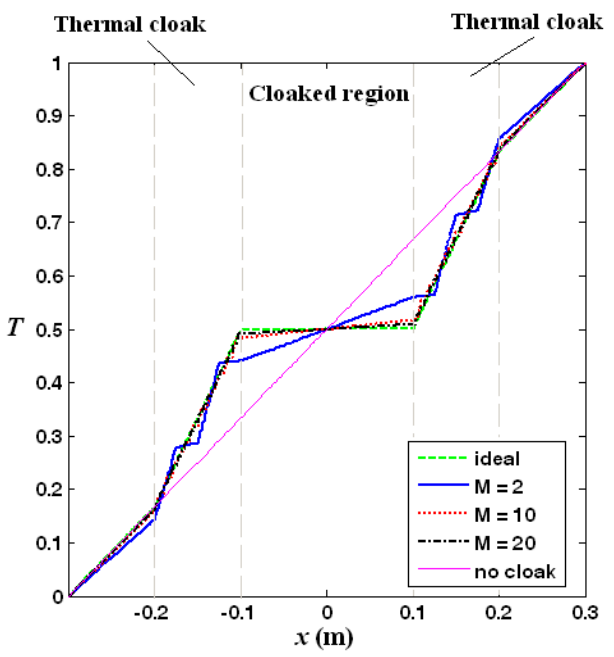

Figure 3: Profiles of the steady-state temperature for the ideal cloak, the segmented cloaks and the case with no cloak.
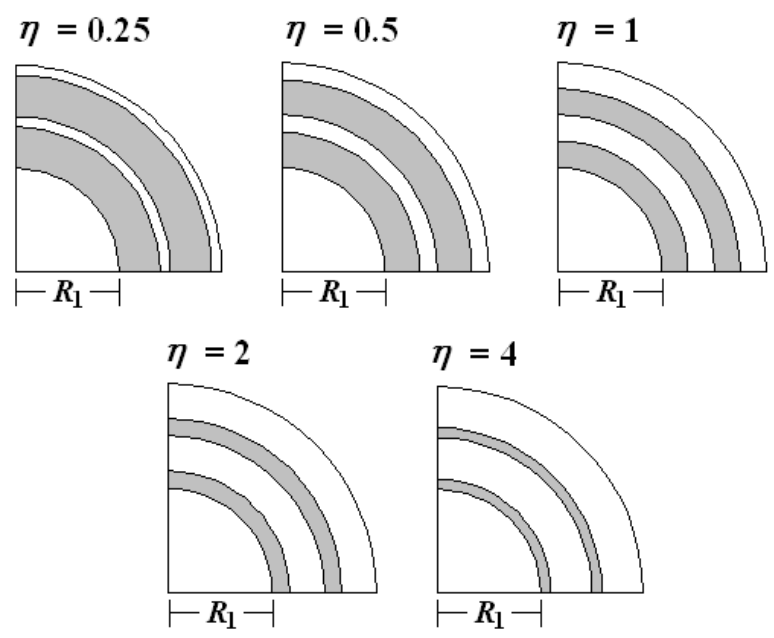

Figure 4: The 2-segmented cloaks with $\eta=0.25,0.5,1,2$ and 4 (sublayer A is shaded in grey).

\subsection{Effects of thickness ratio in the segmented thermal cloak}

The effects of five different thickness ratios were examined, i.e. $\eta=0.25,0.5,1$, 2 and 4. An example of how the different thickness ratio affects the construction of a 2-segmented cloak is illustrated in Figure 4. For thickness ratios of less than 1 , the sublayer with higher thermal conductivity, i.e. sublayer A is thicker than the sublayer with smaller thermal conductivity, i.e. sublayer B, and vice versa. 
Plots of the temperature along the $y=0$ axis of the 2-, 10- and 20-segmented cloaks are shown in Figure 5. The line depicting the ideal cloak case is also shown for comparison. The assessment of the effectiveness of the segmented cloaks is based on the two criteria established in the preceding section.
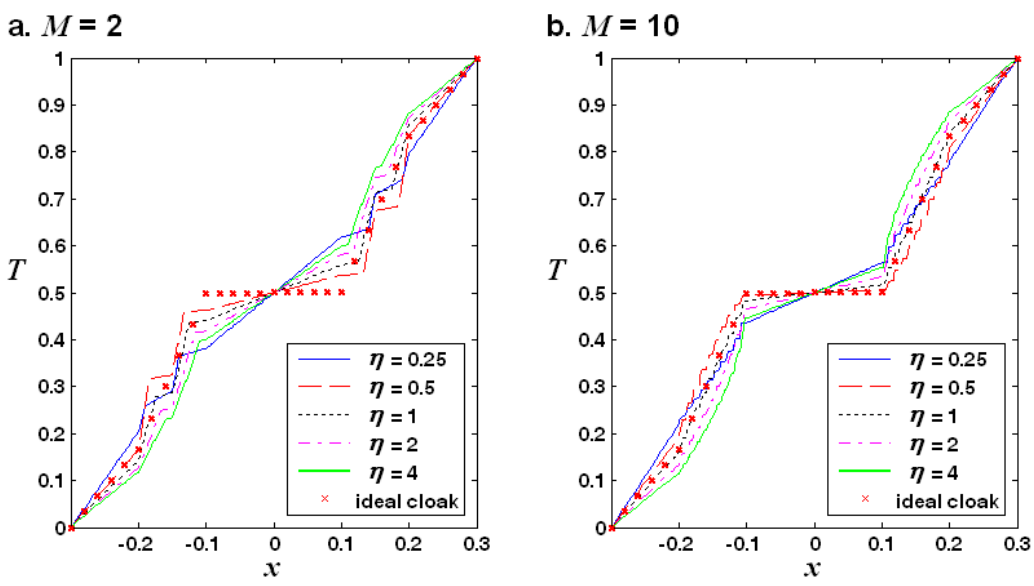

c. $M=20$

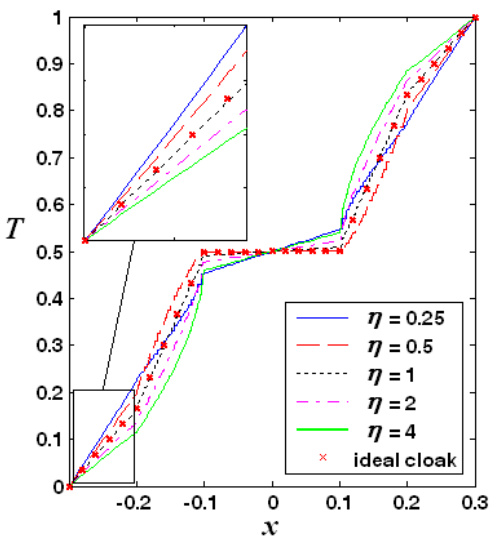

Figure 5: Plots of the temperature profile at the $x$ coordinate $(y=0)$ for the (a) 2- (b) 10- and (c) 20-segmented cloaks for different thickness ratios $\eta$.

Based on the first criterion (ability of the thermal cloak to thermally conceal an object), the best results were found for the segmented cloaks with thickness ratio $\eta=1$, see enlarged view in Figure 5(c). This was observed in the 10- and 20 -segmented cloaks, while the 2-segmented cloak showed the best results for $\eta=0.5$. Based on the second criterion (ability to achieve zero thermal gradient inside the cloaked region), the best results were obtained for the segmented cloaks with thickness ratio of $\eta=0.5$. 
Optimal thermal cloaking cannot be achieved if the thickness ratio is not correctly chosen regardless of the number of segments used. This is indicated by the temperature profiles obtained for $\eta=0.25$ and $\eta=4$ in Figure 5(c), which deviate significantly from the profile of the ideal cloak. The results show that the selection of $\eta$ may not be unique and could depend on whether thermal invisibility or minimum thermal gradient inside the cloaked region should be achieved.

\section{Conclusions}

Numerical results showed that thermal invisibility can be obtained with segmented cloaking. The effectiveness of the segmented cloaks increased with the number of segments used. Segmented cloaking offers a realistic approach in the fabrication of thermal cloaking devices due to the homogeneous and thermally isotropic nature of each sublayer.

The thickness ratio between the sublayers also plays an important role in determining how well the segmented cloak performs. Incorrect choice of the thickness ratio will lead to poor levels of thermal invisibility regardless of the number of segments used. The results from this study showed that thickness ratio of $\eta=1$ produced the best results when comparing the temperature at the outer zone. On the other hand, the thickness ratio of $\eta=0.5$ produced better uniformity of the temperature, i.e. a near zero thermal gradient within the cloaked region.

It is important to point out that the models developed in this study have assumed perfect contact between the adjacent sublayers. In practice, fabrication imperfections may result in discontinuous propagation of heat flux between the two neighbouring sublayers. The effects of the thermal discontinuity between sublayers on the performance of the segmented thermal cloak should be further investigated.

\section{References}

[1] Pendry J.B., Schurig D., Smith D.R., Controlling electromagnetic fields. Science, 2006, 312: 780-782.

[2] Qiu C., Zhang B., Wu B.I., Johnson S.G., Joannopoulos J.D., Spherical cloaking using nonlinear transformations for improved segmentation into concentric isotropic coatings. Optics Express, 2009, 17: 13467-13478.

[3] Wood B., Pendry J.B., Tsai D.P., Directed subwavelength imaging using a layered metal-dielectric system. Physical Review B, 2006, 74: 115116.

[4] Huang Y., Feng Y., Jiang T., Electromagnetic cloaking by layered structure of homogeneous isotropic materials. Optics Express, 2007, 15: 1113311141 .

[5] Schurig D., Mock J.J, Justice B.J., Cummer S.A., Pendry J.B., Starr A.F., Smith D.R., Metamaterial electromagnetic cloak at microwave frequencies. Science, 2006, 314: 977-980. 